\title{
In Vitro Biocompatibility of SiCHA Nanopowders on Human Mesenchymal Stem Cells
}

\author{
Yanny M. Baba Ismail ${ }^{1,2^{*}}$, Ian Wimpenny ${ }^{2}$, Oana Bretcanu ${ }^{3}$, \\ Kenneth W. Dalgarno ${ }^{3}$ and Alicia J. El Haj ${ }^{2}$ \\ ${ }^{1}$ School of Materials and Mineral Resources Engineering, \\ Universiti Sains Malaysia, Engineering Campus, \\ 14300 Nibong Tebal, Pulau Pinang, Malaysia \\ ${ }^{2}$ Institute for Science and Technology in Medicine, Keele University, \\ Stoke-on-Trent, ST47QB, United Kingdom \\ ${ }^{3}$ School of Mechanical and Systems Engineering, Newcastle University, \\ Newcastle-upon-tyne, NE17RU, United Kingdom \\ *Corresponding author: yannymarliana@usm.my
}

Published online: 15 April 2018

To cite this article: Yanny M. Baba Ismail, Ian Wimpenny, Oana Bretcanu, Kenneth W. Dalgarno and Alicia J. El Haj. (2018). In vitro biocompatibility of sicha nanopowders on human mesenchymal stem cells. Journal of Engineering Science, 14: 35-46, https://doi. org/10.21315/jes2018.14.3.

To link to this article: https://doi.org/10.21315/jes2018.14.3

\begin{abstract}
Synthetic hydroxyapatite (HA) possesses good biocompatibility, bioactivity and osteoconductivity and closely mimics the mineralised phase of human bone and teeth. However, for many years the clinical researchers worldwide have been aware that the mineral phase of bone is not solely HA but contains a number of substituents (e.g. $\mathrm{CO}_{3}$, $\mathrm{Si}, \mathrm{Zn}, \mathrm{Sr}, \mathrm{Na}$ and $\mathrm{Mg}$ ). Among the substituents, carbonate is the major substitute ions which are present about 2-8 wt. \% depending mainly on the individual age. The presence of approximately $1 \mathrm{wt}$. \% Si is known to be essential in bone formation and calcification. Thus, the present study is aimed at developing a new biomaterial consisting both of these two biologically important cations into the HA structure by synthesising silicon carbonated HA (SiCHA) powders via nanoemulsion method, and to assess the in vitro biocompatibility of the as-synthesised powders in response to human Bone Marrow derived Mesenchymal Stem Cells (hMSCs). Both the carbonate and silicon ions were successfully substituted into the HA lattice. Powders with carbonate encouraged better cell activity in comparison to the carbonate-free powders. While, SiHA and SiCHA-1 as-synthesised powders with high amount of Si (> $1 \mathrm{wt} . \%)$ tend to kill some cells at the early stage of seeding and hinder cells activity afterwards. Among the tested powders, SiCHA-2 as-synthesised powders is
\end{abstract}


chosen as the optimum composition as it exhibited the highest cell viability and proliferation besides having the closest amount of carbonate and Si ions to the bone mineral.

Keywords: Silicon carbonated hydroxyapatite, nanopowders, human mesenchymal stem cells, in vitro biocompatibility test, bone tissue engineering

\section{INTRODUCTION}

Hydroxyapatite (HA) is among the most widely used bone replacement materials due to its strong affinity with the mineral component of bones; it possesses good bioactivity, osteoconductivity and biocompatibility with the human bone tissue. ${ }^{1,2}$ Although, stoichiometric Hydroxyapatite (HA) $-\mathrm{Ca}_{10}\left(\mathrm{PO}_{4}\right)_{6}$ $(\mathrm{OH})_{2}$ has been a widely used model for the apatite present in the bone tissues for many years, the chemical composition of biological apatites differs from the stoichiometric HA. ${ }^{3}$ The biological apatites are uniquely similar in that they all comprise carbonate in varying amounts of $2-8 \mathrm{wt}$. \%, preferentially substituting the phosphate site (B-type) compared with hydroxyl (A-type) ions in the apatite lattice. The composition of carbonate depends on bone age, site, sex and health of the individual. ${ }^{3,4}$ Among other trace elements present in natural bone, silicon (Si) plays an important role in stimulating bone growth and development. ${ }^{3,5}$

The development of multi-substituted HA powders with a fully controlled level of ionic substitutions into the HA lattice and high similarity to bone mineral, is of great interest to achieve the "gold standard" represented by the natural bone. For this reason, researchers have now focussed on the production of multisubstituted HA for instance, silicon carbonated hydroxyapatites (SiCHA). The adequate solubility of CHA and the benefits of soluble silicon could be combined in order to improve the bioactivity of the apatite bioceramics. ${ }^{4}$ Thus, SiCHA has great potential as a biomedical material for promising approach toward achieving the "gold standard". Several studies on the development of multi-substituted HA powders has been reported in the literature. However, limited studies have investigated the effect of ionic substitutions in the HA lattice on the cell responses. Many research groups focus on the different synthesis techniques used to produce the powders, various physico-chemical characterizations and some solubility tests in simulated body fluid (SBF) to predict the ability to form apatite layer in vitro. ${ }^{6,7}$

Therefore, the aim of this study is to investigate the influences of the ionic substitutions by the incorporation of carbonate and silicon ions simultaneously into the HA structure, and to assess the in vitro biocompatibility of the prepared powders in response to human Bone Marrow derived Mesenchymal Stem Cells (hMSCs). It is crucial to understand the optimum condition (i.e. composition, 
$\mathrm{pH}$, culture condition) required when using the chemically modified HA powders in stimulating optimum cell responses, which leads to the enhancement of the osteogenic activity. In this work, the physico-chemical properties of the prepared powders were investigated through X-Ray Spectroscopy (XPS), Carbon, Hidrogen, Nitrogen $(\mathrm{CHN})$ and Inductive Coupled Plasma-Optical Emission Spectroscopy (ICP-OES) technique. The morphology of the prepared powders was imaged under Transmission Electron Microscope (TEM) and the powders were subsequently tested in vitro with hMSCs via direct contact based on ISO10993-5 in order to inspect the biocompatibility properties.

\section{EXPERIMENTAL}

\subsection{Materials Preparation}

Silicon carbonated $\mathrm{HA}$ (SiCHA) powders were synthesised at room temperature using $\mathrm{Ca}\left(\mathrm{NO}_{3}\right)_{2} \cdot 4 \mathrm{H}_{2} \mathrm{O},\left(\mathrm{NH}_{4}\right)_{2} \mathrm{HPO}_{4}, \mathrm{NH}_{4} \mathrm{HCO}_{3}$ and $\mathrm{Si}\left(\mathrm{CH}_{3} \mathrm{COO}\right)_{4}$ (Sigma-Aldrich, Gillingham, UK) by nanoemulsion method as presented in our previous report. ${ }^{8}$ The amounts of carbonate $(x)$ and silicon $(y)$ substituted into the HA structure (Table 1) were calculated based on the stoichiometry empirical formula as shown below:

$$
\text { SiCHA: } \mathrm{Ca}_{10-\mathrm{x} / 2}\left(\mathrm{PO}_{4}\right)_{6-\mathrm{x}-\mathrm{y}}\left(\mathrm{CO}_{3}\right)_{\mathrm{x}}\left(\mathrm{SiO}_{4}\right)_{\mathrm{y}}(\mathrm{OH})_{2-\mathrm{y}}
$$

Table 1: Amounts of carbonate $(x)$ and silicon $(y)$ substituted into the HA structure.

\begin{tabular}{lcc}
\hline Sample code & Carbonate $(x)$ & Silicon $(y)$ \\
\hline CHA & 2.0 & - \\
SiHA & - & 0.5 \\
SiCHA-1 & 2.0 & 0.5 \\
SiCHA-2 & 2.0 & 0.3 \\
\hline
\end{tabular}

\subsection{Physico-Chemical Characterisations}

\subsubsection{XPS analysis}

XPS is an extremely sensitive technique used to quantify the surface chemistry of the sample at the atomic level. In this study, XPS was used to study the effect of ionic substitutions on the surface atomic chemistry of the prepared powders. This technique required a simple sample preparation where, the powders were formed into a compacted layer (>10 nm thickness) immobilised onto the 
foil which could then be mounted on carbon tape for analysis using Theta Probe instrument (Thermo Scientific, United States). The XPS spectrometer employed a monochromatic Al Ka X-Ray source. Samples were dried completely under ambient conditions before analysis was performed. The XPS analysis was carried out by NEXUS (Newcastle University, United Kingdom). CasaXPS Processing Software was used to analyse the XPS spectrum obtained.

\subsubsection{Quantification of carbonate and silicon substituted in apatite structure}

The percentages of $\mathrm{CO}_{3}$ incorporated in the as-synthesised powders were determined using Carbon, Hydrogen, Nitrogen (CHN) analysis using Carlo Erba 1180 Elemental Analyser controlled with CE Eager 200 Software, run in accordance to the manufacturer's instruction and weighed using a certified Mettler MX5 Microbalance. The powders $(1.5-2.0 \mathrm{mg})$ were combusted at high temperatures in a stream of oxygen, and the products of the combustion for carbon, hydrogen and nitrogen were measured by the instrument in a single analysis. In order to estimate the amount of $\mathrm{CO}_{3}$ present in the sample, the wt. \% of carbon obtain was multiplied by a factor of five.

$0.01 \mathrm{~g}$ of the as-synthesised powders was digested in $1 \mathrm{M} \mathrm{HNO}_{3}(2.5 \mathrm{~mL}$ of $\mathrm{HNO}_{3}, 1.5 \mathrm{~mL}$ of $\mathrm{H}_{2} \mathrm{O}_{2}$, and $0.3 \mathrm{~mL}$ of $\mathrm{HCl}$ ) in a $100 \mathrm{~mL}$ Erlenmeyer flask. The concentrations of $\mathrm{Si}$ in the prepared powders were determined by inductively coupled plasma with optical emission spectroscopy (ICP-OES) using Perkin Elmer Optimal 4300DV instrument. The results obtained was then converted from part per million (ppm) to weight percent (wt. \%).

\subsubsection{Microscopy evaluation}

Philips CM100 Transmission Electron Microscope (TEM) was used to examine the morphology of the as-synthesised powders in terms of their particle size and shape. Prior imaging the samples, $0.1 \mathrm{mg}$ of powders were suspended in pure water and sonicated for $10 \mathrm{~min}$ to allow the powders to be well dispersed. A drop of the suspension was then carefully placed onto a copper grid (diameter $=$ $3.05 \mu \mathrm{m}, \mathrm{mesh}=400)$ and allowed to dry. Samples were imaged at a magnification of $130 \mathrm{kX}$ at $\mathrm{HV}=100.0 \mathrm{kV}$

\subsection{In vitro Biocompatibility Test}

International Organisation for Standardisation (ISO) 10993-5: Biological Evaluation of Medical Devices, Part 5: Tests for Cytotoxicity, was adapted and 
used as guideline in this study. This test involved the study on the cell viability and proliferation of hMSCs in direct contact with the as-synthesised samples.

\subsubsection{Sample preparation}

Aliquots of $0.05 \mathrm{~g}$ of the as-synthesised powders were sterilised in $1.0 \mathrm{~mL}$ of $70 \%$ industrial methylated spirit (IMS) for three hours followed by rinsing twice with Phosphate-buffered saline (PBS; Lonza, United Kingdom).

\subsubsection{Cell culture and seeding}

Human bone marrow derived-mesenchymal stem cells (hMSCs) obtained from a 24-year old male (Lonza, United States) at passage zero (P0) were expanded until passage two (P2) when the required cell number was obtained. Cells were cultured in high glucose expansion media consists of $4.5 \mathrm{~g} / \mathrm{L}$ Dulbecco's Modified Eagle Medium, DMEM (Lonza, United Kingdom), 1\% v/v L-glutamine (Lonza, United Kingdom), 1\% v/v Penicilin-Streptomycin (Lonza, United Kingdom) and 10\% v/v Fetal Bovine Serum (Biosera labtech, United Kingdom) followed by, incubation at humidified environment at $37^{\circ} \mathrm{C}$ with $5 \% \mathrm{CO}_{2} . \mathrm{hMSCs}$ at passage 3 (P3) were seeded at $5 \times 10^{4}$ per well in a 24 well cell culture plate and allowed to adhere for three hours. Prior to direct contact of the powders with the seeded cells, $1.0 \mathrm{~mL}$ of osteogenic media was directly added into the aliquots containing $0.05 \mathrm{~g}$ of sterile powders, mixed well and carefully transferred to the relevant wells. Culture media was replenished every three days for 14 days. In all cases, tissue culture plastic alone cultured in osteogenic media acts as the positive control (non-toxic) whilst negative control (toxic) consists of $0.1 \%$ Triton-X in high glucose expansion media. At seven and 14 days, cells were rinsed with Phosphate Buffer Saline, PBS (Sigma-Aldrich, United Kingdom), trypsinised, washed again with PBS and samples were lysed in $1.0 \mathrm{~mL}$ of $\mathrm{dH}_{2} \mathrm{O}$ followed by being frozen at $-80^{\circ} \mathrm{C}$.

\subsubsection{Cell viability}

The cell viability was observed using Confocal Laser Scanning Microscope (CLSM) Olympus Fluoview FV 1200 with Fluoview Version 4.1 software (Olympus, UK). The viability of the cells was assessed at 14 days using the Live/ Dead Assay Kit (Invitrogen, United Kingdom) according to the manufacturer's instructions. Calcein-AM ester was used to fluorescently label viable cells (green); the nucleus of dead cells is labelled with Propidium Iodide (red). Briefly, cell culture media was removed from samples. They were washed with $1.0 \mathrm{~mL}$ PBS then immersed in $0.5 \mathrm{~mL}$ PBS staining solution containing $10 \mu \mathrm{M}$ Calcein-AM 
and $1 \mu \mathrm{M}$ Propidium Iodide and incubated at $37^{\circ} \mathrm{C}$ for $20 \mathrm{~min}$ in the dark. The samples $(n=1)$ were then washed once with $1.0 \mathrm{~mL}$ of PBS and immediately imaged using CLSM.

\subsubsection{Cell Proliferation}

The Quant-iT ${ }^{\mathrm{TM}}$ Picogreen ${ }^{\circledR}$ dsDNA assay kit (Invitrogen, United Kingdom) was used according to the manufacturer's instruction. The Picogreen solution was prepared as 1:200 dilutions in 1× Tris-EDTA (TE) buffer. Ranges of DNA dilutions $(0-2 \mu \mathrm{g} / \mathrm{mL})$ were used to construct a standard curve. $100 \mu \mathrm{L}$ of cell lysate or DNA standard was placed each well of a 96 well plate, followed by $100 \mu \mathrm{L}$ of Picogreen reagent to each well. This was placed in the dark for five min before reading the fluorescence at 485/535 $\mathrm{nm}$ (excitation/emission) using Synergy II BioTek plate reader.

\subsubsection{Statistical analysis}

Quantitative data were presented as means \pm standard deviation (SD). Data were initially tested for normality using the Kolmogorov-Smirnov test, with Dallal-Wilkinson-Lillie for corrected $P$ value. To determine any differences between powders group at each time point, a two-way ANOVA with multiple comparisons Tukey test was performed. Statistical significance was considered for $p \leq 0.05(*), p \leq 0.01(* *), p \leq 0.001(* * *)$ and $p \leq 0.0001(* * * *)$. For biochemical assays, tests were performed on $n=3$ in duplicate. All statistical analyses were performed using GraphPad Prism 7 software.

\section{RESULTS AND DISCUSSION}

\subsection{Physico-chemical Properties}

XPS was used to confirm the surface atomic composition of the assynthesised powders. The XPS spectrum for the as-synthesised powders is shown in Figure 1, demonstrating the elemental peaks of Ca2p, P2p, C1s, Si2p and O1s at the typical binding energies of 349, 135, 209, 101 and $533 \mathrm{eV}$, respectively. ${ }^{9}, 10$ Results indicated that both carbonate and silicon ions were successfully substituted into the HA lattice.

The percentage of carbonate present in the apatite structure was found to be in the range of carbonate typically present in bone mineral, which is 2-8 wt. \%. ${ }^{1,11}$ Among the as-synthesised powders, the highest amount of carbonate 
was detected in CHA with 7.4 wt. \%, followed by 3.9 wt. \% in SiCHA-1 and 5.2 wt. \% in SiCHA-2 (Table 2). Minor amount of carbonate was detected in SiHA as-synthesized powders (carbonate free powders), due to the absorption of carbon gas from the atmosphere during synthesis. The presence of carbonate ions was reported to have positive impacts on both bioactivity as well as mechanical properties. Higher carbonate contents leads to higher metabolic activity as suggested in the literature. ${ }^{3,11}$ In addition, the substitution of carbonate in the HA structure has also showed improved mechanical properties relative to pure HA as shown in our previous finding. ${ }^{12}$

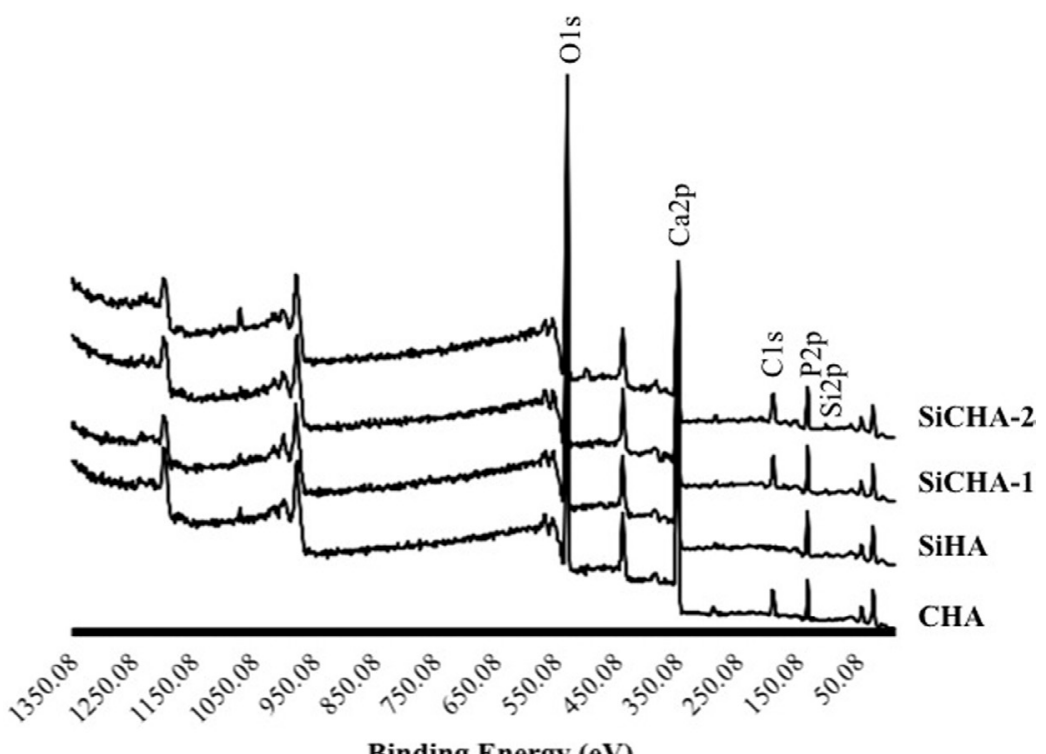

Binding Energy (eV)

Figure 1: XPS survey spectra for CHA, SiHA, SiCHA-1 and SiCHA-2 nanopowders. Fitting analysis of the Ca2p, P2p, Si2p, C1s and O1s spectra confirmed the presents of different functional groups on the surface.

ICP-OES is a chemical analysis, which was performed on SiCHA assynthesised powders to confirm the presence of silicon ions. The results suggested that the amount of Si presents was relatively high as compared to the amount of Si found in vivo within the mineralising osteoid regions, which is $<1.0 \mathrm{wt}$. \% (Table 2). Several in vitro and in vivo studies have shown the role of Si for bone growth development. The presence of Si ions could influence the biomineralisation process and has a dose-dependent effect on the cell activities and collagen synthesis. ${ }^{1,5}$ It is critically important to control the amount of Si present in the apatite structure as high amount of Si was reported to induce a toxic effect. ${ }^{3}$ 
Table 2: Percentages of carbonate (wt. $\% \mathrm{CO}_{3}$ ) and silicon ( $\left.\mathrm{Si}\right)$.

\begin{tabular}{lcc}
\hline Sample code & Carbonate $\left(\right.$ wt. $\left.\% \mathrm{CO}_{3}\right)$ & Silicon (wt. \%) \\
\hline CHA & 7.4 & - \\
SiHA & - & 1.5 \\
SiCHA-1 & 3.9 & 1.2 \\
SiCHA-2 & 5.2 & 0.8 \\
\hline
\end{tabular}

Regardless of their compositions, all of the as-synthesised powders revealed a rod-like morphology (Figure 2). The particle sizes of the as-synthesised powders fall in the range of $30-50 \mathrm{~nm}$ in length and $10-20 \mathrm{~nm}$ wide, which is similar to the dimensions of biological apatite. ${ }^{13}$ Producing nanoscale powders is critically important in particular for bone tissue engineering applications as to provide better bioactivity and most importantly as to closely mimic the architecture of natural bone and teeth. ${ }^{14,15}$
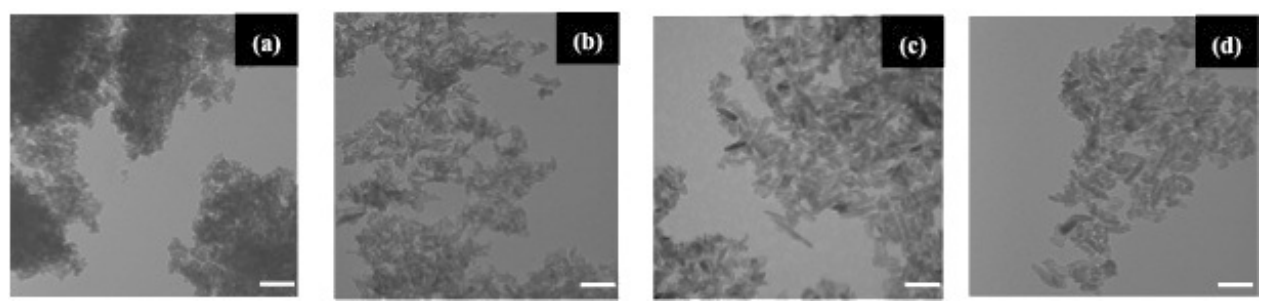

Figure 2: TEM micrographs of as-synthesised (a) CHA, (b) SiHA, (c) SiCHA-1 and (d) SiCHA-2 powders (White scale bar $=100 \mathrm{~nm}$ ).

In general, Live/dead staining of hMSCs in direct contact with the assynthesised powders after 14 days in culture showed that all powders are considered biocompatible to hMSC cells (Figure 3). Majority of the cells remained viable, indicated by the green fluorescence. However, cells in direct contact with the tested powders containing higher amount of $\mathrm{Si}$, particularly SiHA and SiCHA-1 showed lower proportion of viable cells as compared to CHA and SiCHA-2. It was also observed that there was colour changed of the culture media from red to orange as the culture period prolonged (data not shown). The $\mathrm{pH}$ of the culture media was also dropped to about $\mathrm{pH} 5.8$, which is relatively lower and slightly acidic as compared to optimum $\mathrm{pH}$ of culture media required for mammalian cell growth that is approximately $\mathrm{pH}$ 7.2. It is found that higher amount of Si (>1 wt. \%) induced slightly toxic culture condition where it has killed some amount of cells at the early stage of culture and slower the activity of the remaining cells. Similar observation was reported in the literature. ${ }^{3}$ This can be explained by the ionic dissociation from the main structure into the surrounding environment. It is know 
that higher Si leads to higher solubility of the powders. Thus, it is easier for the ions to dissociate once in contact with the culture media and immediately attacked the seeded cells. As a result from high ions released, some of the cells were detached from the powders at the initial seeding period, floating in the culture media and subsequently caused cell death as being an anchorage-dependent cells, hMSCs need a substrate to be attached. ${ }^{8}$ In order to overcome this problem, heat treatment of the powders such as calcination could be performed in order to ensure the ions are properly substituted into the apatite structure and hence could improve the overall performances of the powders.
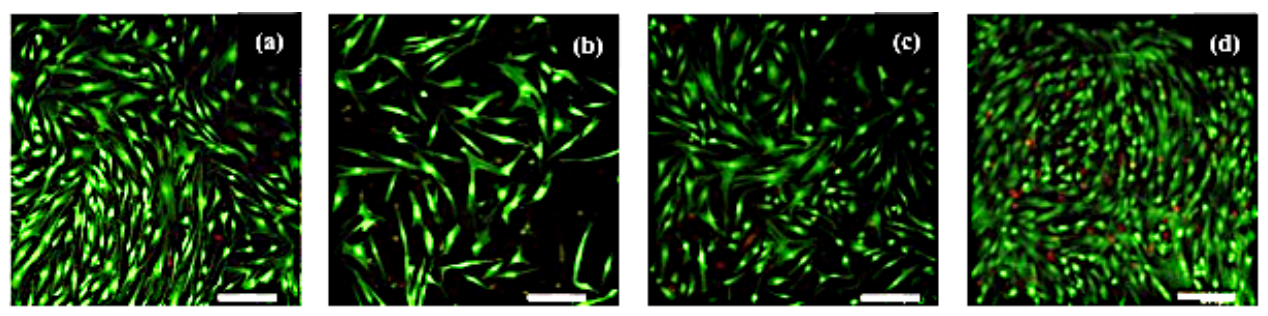

Figure 3: Cell viability of hMSCs in direct contact with as-synthesised (a) CHA, (b) SiHA, (c) SiCHA-1 and (d) SiCHA-2 powders after 14 days in culture (White scale bar $=200 \mathrm{~nm}$ ).

DNA concentration was used to indicate the cell population. Results supported the observation of cell viability, identifying that CHA outperformed the other tested powders at all time-points in terms of DNA quantity and viability, followed closely by SiCHA-2. However, generally all materials encouraged cell proliferation. hMSCs were found to show greatest level of cell proliferation on materials containing both carbonate and silicon substituted into the HA structure simultaneously as compared to carbonate-free materials. This negative behaviour of SiCHA-1 and SiHA were apparently in contrast with the previous study reported in the literature, ${ }^{16,17}$ where good interaction of human osteoblasts was found as in contact with high Si-substituted HA (0.8 wt. \%). The discrepancy in the results is due to different nature of the tested materials. For instance, the work conducted by Botelho et al. (2006) was based on dense, sintered SiHA pellets. ${ }^{16}$ Thus, the solubility-reactivity and the extent of the biomaterial-cell interactive surface were remarkably lower as compared to that of a powdered form. ${ }^{3}$ 


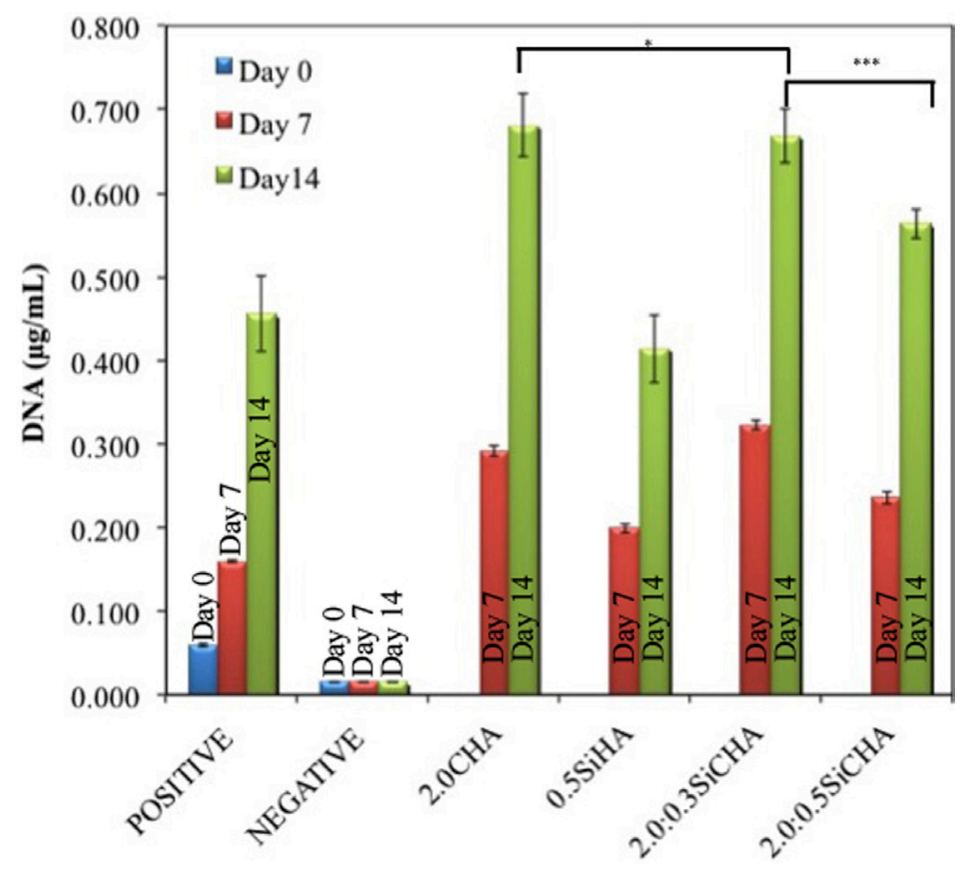

Figure 4: Effect of carbonate and silicon ions substituted into HA structure on cell proliferation at 7 and 14 days of culture on hMSCs. Positive control represents tissue culture plastic (TCP) in osteogenic media, while cells cultured in $0.1 \%$ Triton- $\mathrm{X}$ in osteogenic media denoted as the negative control. Values represent the mean $\pm \mathrm{SD}$ of three samples in duplicate $(* p \leq 0.05, * * p \leq 0.01$, $* * * p \leq 0.001, * * * * p \leq 0.0001)$.

\section{CONCLUSIONS}

1. Controlled amounts of ionic substitutions into the apatite structure play an important role in determining the cell fate, as the solubilityreactivity and biomaterial-cell interactive surface (mainly because of ionic substitutions, absence of heat treatment and nano-size powders) are the two major factors, which define cell survival and response.

2. Excessive incorporation of $\mathrm{Si}$ ions ( $>1 \mathrm{wt}$. \%) into the apatite structure hindered cell proliferation and at some extents could cause cell death.

3. Powders produced in this work have high potential to be used in the fabrication of scaffolds, coating materials as well as injectable bona paste for Bone Tissue Engineering (BTE) applications. 


\section{ACKNOWLEDGEMENTS}

This research was supported by the Arthritis Research UK Tissue Engineering Centre (ARUK-TEC Award 19429, Grant No. R5242-B005) and the Ministry of Higher Education Malaysia (Grant No. R5240-B273). We would like to thank the National EPSRC XPS Users Service (NEXUS), Newcastle University for conducting the XPS analysis.

\section{REFERENCES}

1. Sprio, S. et al. (2008). Physico-chemical properties and solubility behaviour of multi-substituted hydroxyapatite powders containing silicon. Mater. Sci. Eng. C, 28(1), 179-187, https://doi.org/10.1016/j.msec.2006.11.009.

2. Marchat, D. et al. (2013). Physico-chemical characterization and in vitro biological evaluation of pure SiHA for bone tissue engineering application. Key Eng. Mater., 529-530, 351-356.

3. Landi, E. et al. (2010). Human osteoblast behavior on as-synthesized $\mathrm{SiO}(4)$ and $\mathrm{B}-\mathrm{CO}(3)$ co-substituted apatite. J. Biomed. Mater. Res. A, 94, 59-70, https://doi.org/10.1002/jbm.a.32671.

4. Boyer, A. et al. (2012). Synthesis and characterization of Cx-Siy-HA for bone tissue engineering application. Key Eng. Mater., 529-530, 100-104, https://doi.org/10.4028/www.scientific.net/KEM.529-530.100.

5. Hing, K. A. et al. (2006). Effect of silicon level on rate, quality and progression of bone healing within silicate-substituted porous hydroxyapatite scaffolds. Biomaterials, 27(29), 5014-5026, https://doi. org/10.1016/j.biomaterials.2006.05.039.

6. Boanini, E., Gazzano, M. \& Bigi, A. (2010). Ionic substitutions in calcium phosphates synthesized at low temperature. Acta Biomater, 6(6), 18821894, https://doi.org/10.1016/j.actbio.2009.12.041.

7. Marchat, D. et al. (2013). Accurate characterization of pure siliconsubstituted hydroxyapatite powders synthesized by a new precipitation route. Acta Biomater., 9, 6992-7004, https://doi.org/10.1016/j.actbio. 2013.03.011.

8. Baba Ismail, Y. M. et al. (2017). Development of multisubstituted hydroxyapatite nanopowders as biomedical materials for bone tissue engineering applications. J. Biomed. Mater. Res. Part A, 105(6), 17751785, https://doi.org/10.1002/jbm.a.36038.

9. Zhao, M. Y. et al. (2014). LBL coating of type I collagen and hyaluronic acid on aminolyzed PLLA to enhance the cell-material interaction. Express Polym. Lett., 8(5), 322-335, https://doi.org/10.3144/ expresspolymlett.2014.36. 
10. Kim, T. G. et al. (2010). Microstructured scaffold coated with hydroxyapatite/collagen nanocomposite multilayer for enhanced osteogenic induction of human mesenchymal stem cells. J. Mater. Chem., 20, 8927-8933.

11. Landi, E. et al. (2003). Carbonated hydroxyapatite as bone substitute. J. Eur. Ceram. Soc., 23(15), 2931-2937, https://doi.org/10.1016/S09552219(03)00304-2.

12. Baba Ismail, Y. M. \& Mohd Noor, A. F. (2011). Effect of a novel approach of sintering on physical properties of carbonated hydroxyapatite. J. Mater. Sci. Eng. B, 1, 157-163.

13. Wang, J. \& Shaw, L. L. (2009). Nanocrystalline hydroxyapatite with simultaneous enhancements in hardness and toughness. Biomaterials, 30(34), 6565-6572, https://doi.org/10.1016/j.biomaterials.2009.08.048.

14. Dorozhkin, S. V. (2009). Calcium orthophosphates in nature, biology and medicine. Materials, 2, 399-498, https://doi.org/10.3390/ma2020399.

15. Dorozhkin, S. V. (2012). Nanodimensional and nanocrystalline calcium orthophosphates. Am. J. Biomed. Eng., 2(3), 48-97, https://doi. org/10.5923/j.ajbe.20120203.01.

16. Botelho, C. M. et al. (2006). Human osteoblast response to siliconsubstituted hydroxyapatite. J. Biomed. Mater. Res., 79A(3), 723-730, https://doi.org/10.1002/jbm.a.30806.

17. Pietak, A. M. et al. (2007). Silicon substitution in the calcium phosphate bioceramics. Biomaterials, 28(28), 4023-4032, https://doi.org/10.1016/j. biomaterials.2007.05.003. 